\title{
Treatment of uveitis with recombinant human interleukin-13
}

François G Roberge, Marc D de Smet, Jacques Benichou, Martin F Kriete, James Raber, John Hakimi sis of the proinflammatory cytokines IL-1, IL-6, IL-8, tumour necrosis factor $\alpha(\mathrm{TNF}-\alpha)$, and macrophage inflammatory protein $1 \alpha$ $(\mathrm{MIP}-1 \alpha) \cdot{ }^{4-7}$ Conversely, the release of the IL-1 receptor antagonist is stimulated. ${ }^{5}$ IL-13 also suppresses the production of nitric oxide as well as the induction of cyclo-oxygenase- 2 in macrophages. ${ }^{689}$ In the bone marrow, IL-13 enhances the proliferation and differentiation of haemopoietic stem cells, and was reported to induce a marked monocytosis in mice..$^{11}$ IL-13 does not directly affect T cells. However, by inhibiting the production of IL-12 and interferon $\alpha$, IL-13 may prevent the development of the Th-1 lymphocyte pathway which seems determinant for most posterior uveitis. ${ }^{5}{ }^{12}$ Thus, the anti-inflammatory properties of IL-13 suggested that it could be a powerful new agent for the treatment of uveitis. We evaluated the efficacy of human IL-13 in monkeys to which uveitis was induced by immunisation with the retinal S-antigen. In addition, we monitored the effect of IL-13 on the blood leucocyte levels. effect of IL-13 extended into the 4 week follow up period. IL-13 produced an increase in circulating polymorphonuclear neutrophils and a decrease in lymphocytes.

National Institutes of Health, National Eye Institute, Bethesda, Maryland, USA F G Roberge

M D de Smet M F Kriete

J Raber

National Institutes of Health, National Cancer Institute, Biostatistics Branch, Bethesda, Maryland, USA

J Benichou

Roche Research Center, Inflammation and Autoimmune Diseases,

Hoffmann-LaRoche, Nutley, New Jersey,

USA

J Hakimi

Correspondence to: François G Roberge, MD, La Pitié-Salpêtrière Hospital, Department of

Ophthalmology, 47-83 Blvd de l'Hôpital, Bât Babinski, 75013 Paris, France.

Accepted for publication 11 March 1998
Conclusion-Administration of IL-13 appears to be a promising modality of treatment for severe uveitis.

(Br f Ophthalmol 1998;82:1195-1198)

The treatment of severe non-infectious uveitis is often based on the broad anti-inflammatory properties of corticosteroids. These properties are, however, counterbalanced by serious metabolic side effects. ${ }^{1}$ In order to reduce this toxicity, the steroids are increasingly used at lower doses in combination with other cytostatic and cytotoxic drugs. ${ }^{3}$ Unfortunately, each drug has its own toxic side effects. Clearly, the goals of treatment would be better achieved with a therapeutic agent possessing a spectrum of activity comparable with corticosteroids but with reduced side effects. In the last few years, cytokines such as interleukin-10 (IL-10) and transforming growth factor $\beta$ (TGF- $\beta$ ) have attracted attention because of their multifaceted downregulating effect on the activity of the immune system. More recently, the newly discovered cytokine IL-13 was found to have even more promising anti-inflammatory effects. $^{4}$

IL-13 is a pleiotropic cytokine produced by Th-2 lymphocytes. ${ }^{4}$ IL-13 shares with the glucocorticoids a downregulating effect on cell mediated immunity. IL-13 inhibits the synthe-

\section{Materials and methods}

INDUCTION OF UVEITIS

The animals participating in the experiment were female cynomolgus monkeys, Macaca fascicularis, weighing between 2.5 and $3.0 \mathrm{~kg}$. All were seronegative for the following viruses: SIV, SRV-1, SRV-2, SRV-5, and STLV-1. Before immunisation, a baseline serum sample was collected, and the normality of the eyes was verified by indirect ophthalmoscopy and pressure tonometry. The animals were immunised with $40 \mu \mathrm{g} / \mathrm{kg}$ of recombinant human S-antigen solubilised at $1 \mathrm{mg} / \mathrm{ml}$ in phosphate buffered normal saline solution (PBS), $\mathrm{pH}$ 7.3, emulsified 1:1 v:v in TiterMax adjuvant (CytRx Corporation, Norcross, GA, USA), and injected subcutaneously at four sites in the nape of the neck. The immunisation, as well as subsequent examinations, was done with the animals anaesthetised by intramuscular injections of ketamine (Aveco, Forth Dodge, IA, USA) $10-20 \mathrm{mg} / \mathrm{kg}$, and atropine (Astra Pharmaceutical, Westboro, MA, USA) $0.05 \mathrm{mg} / \mathrm{kg}$. The principles of laboratory animal care of NIH publication No 86-23, revised in 1985, were followed.

TREATMENT PROTOCOL

From 14 days after immunisation, the animals were examined every 3 and 4 days alternatively by indirect ophthalmoscopy. The pupils were dilated with topical atropine sulphate $1 \%$ solution (Akorn Inc, Abita Spring, LA, USA). 
Treatment was initiated at the first sign of intraocular inflammation (see below). The animals were randomly assigned to two treatment groups of five. The treatment consisted of recombinant human IL-13 at $25 \mu \mathrm{g} / \mathrm{kg}$ in 0.2 $\mathrm{ml}$ PBS, or PBS alone, injected subcutaneously once a day for 28 days. (IL-13 was provided by Hoffmann-LaRoche Inc, Nutley, NJ, USA, under licence from Sanofi-Recherche, Paris, France.) The dose of IL-13 was determined in preliminary toxicological and pharmacokinetic assays at Sanofi and Roche respectively, indicating safety at that dose and an effective serum level as estimated on the TF- 1 cell assay in vitro. After the treatment period, the animals were followed for an additional 28 days.

DISEASE EVALUATION

The intraocular inflammation was evaluated by indirect ophthalmoscopy on an alternate 3 and 4 day schedule and scored as follows, according to the published grading of Guex-Crosier et $a l .{ }^{13}$ The vitreous haziness was graded on a scale of 0.0 to 4.0 by reference to a standardised panel of ocular fundus photographs as described. ${ }^{14}$ Retinal and choroidal inflammation were each graded from 0.0 to 4.0 , based on areas of cellular infiltration as follows: the fundus was divided into four quadrants centred on the optic nerve head; the presence of leucocyte infiltration was scored as 1.0 point per involved quadrant. Similarly, the presence of intraretinal haemorrhages was scored as 1.0 point per quadrant. While retinal vasculitis was also scored by quadrant, the grading was adjusted for partial segmental vascular sheathing or continuous sheathing scored 0.5 and 1.0 point respectively. The sum from 0 to 20 of these scores $(0.0$ to 4.0 for inflammation in the vitreous, retina, choroid, vascular sheathing, and retinal haemorrhages), constituted the grade for each eye. If the vitreous haziness reached levels precluding evaluation of the fundus, the score of the fundus for this time point was derived as the average of the previous

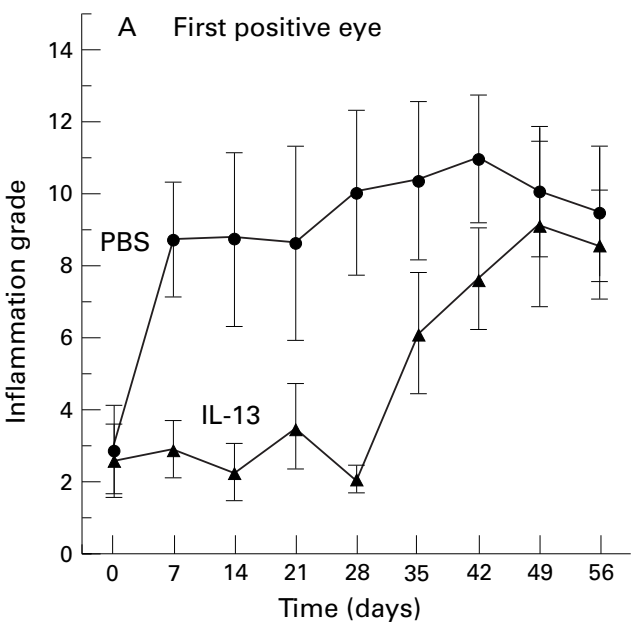

- IL-13 treatment $\rightarrow$ Follow up $\longrightarrow$ score with the first score following the loss of visibility of the fundus. The scoring examiner could not be blinded to the treatment group; but the scoring was limited to a simple choice of presence or absence of each sign to avoid estimation bias. Additional examination consisted in blood samples collection for the determination of leucocyte differential count by automatic cytometry (Coulter Corp, Miami, FL, USA) at the beginning and end of the treatment period.

CYTOKINE LEVEL MEASUREMENT IN THE BLOOD One $\mathrm{ml}$ of serum was collected on the day of immunisation and at 1 week intervals from the day of disease onset, and stored at $-70^{\circ} \mathrm{C}$. The levels of the following cytokines were evaluated with commercial human cytokine assay kits: IL-1 $\beta$, IL-12, and TNF- $\alpha$ (R+D Systems, Minneapolis, MN, USA); IL-2 (DuPont NEN, Boston, MA, USA); and IL-6 (Endogen, Cambridge, MA, USA).

STATISTICAL ANALYSIS OF UVEITIS EXPERIMENT A linear model with normal error was used, with inflammation grade (from 0 to 20 ) as the outcome variable. The model included the following covariates: treatment group (two groups, PBS and IL-13), time (eight time points after initiation of treatment), and initial inflammatory grade at time point zero. The latter term was introduced in order to control for individual differences among animals. Separate models were fitted to the first and second positive eyes. An average overall treatment effect (that is, over the entire time range of the experiment) was estimated from the model and tested with an $\mathrm{F}$ test, through a least squares analysis. We used the procedure GLM of SAS. ${ }^{15}$

\section{Results}

The disease was initially expressed unilaterally for at least 7 days in all animals, except one in the IL-13 treated group which presented with

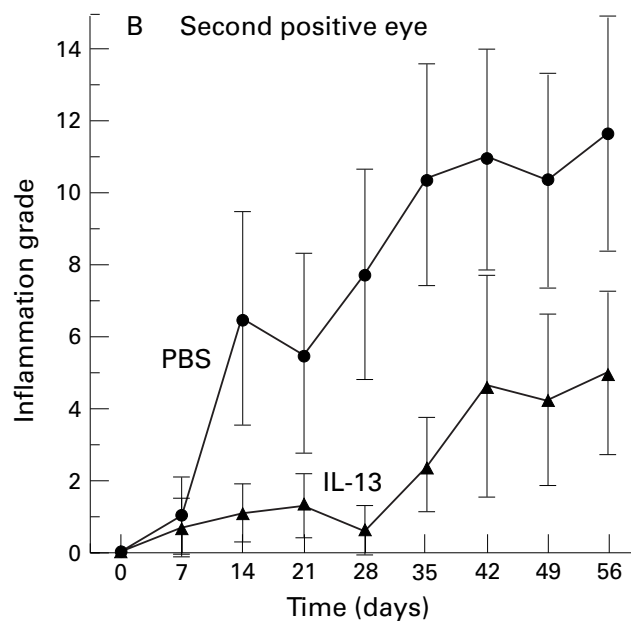

- IL-13 treatment $\rightarrow-$ Follow up $\longrightarrow$

Figure 1 Inhibition of uveitis with IL-13. Uveitis was induced by immunisation with retinal S-antigen. Two groups of five monkeys were treated with IL-13 (25 $\mathrm{\mu g} / \mathrm{kg})$, or vehicle control, by subcutaneous injection once a day for 28 days, and followed for an additional 28 days. Uveitis was detected in one eye at least 7 days before the fellow eye in nine of 10 animals. The results are given separately for the eyes in which uveitis had already started $(A)$, and for the eyes that were negative or with the least severe disease at the time of treatment initiation $(B)$; they represent the mean inflammation score $( \pm S E M)$. 
Table 1 Effect of IL-13 treatment on blood leucocyte levels ( \pm SEM)

\begin{tabular}{llllll}
\hline Normal range & $\begin{array}{l}\text { Time } \\
\text { point }\end{array}$ & $\begin{array}{l}\text { Total } \\
7-15 \times 10^{6} / \mu l\end{array}$ & $\begin{array}{l}\text { PMN } \\
30-55 \%\end{array}$ & $\begin{array}{l}\text { Lymphocytes } \\
35-70 \%\end{array}$ & $\begin{array}{l}\text { Monocytes } \\
1-5 \%\end{array}$ \\
\hline Animal groups: & & & & & \\
Controls & Day 0 & $6.96(0.61)$ & $46.2(3.2)$ & $46.0(1.9)$ & $3.0(0.7)$ \\
IL-13 treated & Day 28 & $8.42(1.25)$ & $45.4(9.2)$ & $45.0(8.7)$ & $4.2(1.0)$ \\
& Day 0 & $8.73(1.71)$ & $51.7(12.0)$ & $41.7(11.4)$ & $1.7(0.8)$ \\
& Day 28 & $13.1(2.21)$ & $66.2(6.1)$ & $29.2(5.8)$ & $1.7(0.8)$ \\
\hline
\end{tabular}

Blood samples were collected on the day of treatment initiation (day 0 ) and at the end of the treatment period (day 28). Blood leucocytes numbers were determined with an automatic Coulter counter.

an asymmetrical bilateral inflammation minimal in one eye (one small spot of sheathing on a retinal vessel). Since the treatment was instituted at the onset of disease, the results are reported separately for the eyes already inflamed when it was started, and for the eyes that were negative or with the least amount of inflammation for the one animal with bilateral disease. In both eyes, the treatment with IL-13 significantly reduced the severity of uveitis compared with controls. The effect was pronounced both in the eyes in which the disease started (Fig 1A), where the average estimated reduction in the inflammatory grade was $6.0(p=0.0001)$, and in the eyes with no or minimal disease at treatment initiation (Fig $1 \mathrm{~B})$, where the estimated average reduction was $3.7(p=0.0001)$. In the eyes that were free of disease when the treatment started, uveitis induction was almost completely inhibited by IL-13. Of these four eyes, three were still negative at the end of the treatment period, while four of the five initially negative eyes of the control group were positive for experimental autoimmune uveitis (EAU). After cessation of therapy, there was a progressive increase of inflammation in the IL-13 treated group. However, the beneficial effect of IL-13 treatment was still observed during the 4 week follow up period. The inflammation score over that period was significantly lower in the IL-13 group in both eyes $(p=0.038$ and $p=0.0001$ in the first and second positive eyes respectively).

In the IL-13 treated animals, there was an increase in the total number of circulating leucocytes (Table 1). The differential count showed a proportional increase of the polymorphonuclear neutrophils (PMN) above the normal range, and a decrease of lymphocytes below the normal range. The differences could not be reliably assessed statistically (by nonparametric paired data test) because of the low number of data points. We observed no effect on the proportion of monocytes.

The serum levels of the cytokines IL- $1 \beta$, IL-2, IL-6, IL-12, and TNF- $\alpha$ were assayed by enzyme linked immunosorbent assay (ELISA) before immunisation, and at 1 week interval during the treatment period. The levels of these cytokines did not rise above the detection limit of the assays used (data not shown).

\section{Discussion}

Treatment with IL-13 resulted in a dramatic improvement of the inflammatory signs of uveitis. The inflammation was inhibited during the entire treatment period. After cessation of treatment, disease progression tended to resume. No signs of toxicity were observed systemically or at the sites of injection. We paid particular attention to the induction of monocytosis. It has been reported that in mice infused continually for 7 days by means of an osmotic pump with high doses of murine IL-13, a 10-fold increase in the number of circulating monocytes was induced. ${ }^{11}$ In our animals, such an effect was not encountered after 1 month of subcutaneous injections.

An aspect of IL-13 treatment, that is not completely reflected in the global scoring, is the rapid improvement of specific variables of inflammation. The change was most notable in perivasculitis, where severe continuous sheathing of a vascular arcade completely disappeared within 1 week of treatment in some animals. The inhibitory effect of IL-13 on the production of IL- 1 , IL- 6 , and TNF- $\alpha$, which serve to activate the vascular endothelial cells, may explain this observation. The activated vascular endothelium normally produces chemokines such as IL-8 and RANTES to attract macrophages and polymorphonuclear neutrophils to inflammatory sites. The inhibitory effect of IL-13 on the production of these two chemokines is thus probably an important aspect of its anti-inflammatory action in uveitis, and may explain the reduction of the retinal vascular sheathing. ${ }^{16}$

In vivo, the inflammatory activity of cytokines results from a complex interaction in which they often stimulate the production of each other and exert combined synergistic effects. For example TNF- $\alpha$ induces IL- 6 and IL-1, and conversely both IL- 6 and IL- 1 can stimulate TNF- $\alpha$ secretion. ${ }^{17}$ At the present time, there are limited specific data on the role played by cytokines in human intraocular inflammation. ${ }^{18}{ }^{19}$ However a vast body of experimental observations indicate an important involvement of IL-1, IL-6, IL-8, and TNF- $\alpha$ in uveitis. ${ }^{20-23}$ Various inflammatory conditions tend to go through common pathways using these cytokines. Thus, the broad inhibitory activity of IL-13 could be advantageous in the treatment of several types of uveitis.

There are also strong indications that $\mathrm{T}$ cells are central to the mechanism of posterior uveitis. ${ }^{24-27}$ Indeed, a majority of these uveitis can be improved by treatment with anti-T cell drugs such as cyclosporin A and FK506. ${ }^{38} \mathrm{~T}$ cells do not have receptors for IL-13, but the modification of the cytokine profile induced by IL-13 appears to shift the immune response away from the $\mathrm{T}$ cell directed delayed type hypersensitivity reaction. ${ }^{12} 29$ In this type of inflammation, the macrophage is the principal effector cell under the direction of Th-1 cells. IL-13 by deactivating several macrophage functions can prevent tissue damage. ${ }^{56}$ Such an effect has been observed in a rat model of experimental autoimmune encephalitis in which the brain damage was considerably reduced in rats that had been injected with cells secreting IL-13. ${ }^{30}$ In the present study, the fact that EAU resumed its active progression soon after stopping IL-13 injections points in the direction of an inhibition of the efferent 
phase of the DTH reaction, without much alteration in the $\mathrm{T}$ cells acquired anti-S-Ag cognitive reactivity.

In the past, the inhibitory effect of drugs in the animal model of experimental autoimmune uveitis has been reliably predictive of their therapeutic effect in humans. Contrary to most biological peptides used in therapeutic trials to date, IL-13 did not have to be given intravenously, but was effective by a simple subcutaneous injection once a day. This mode of administration, commonly used for insulin treatment in diabetes, is more readily accepted by patients. Overall, the results that we obtained in subhuman primates certainly support further study of IL-13 in view of its use in human uveitis.

1 Rubin B, Palestine AG. Complications of corticosteroids and immunosuppressive drugs. Int Ophthalmol Clin 1989;29:159-71.

2 Towler HM, Whiting PH, Forrester JV. Combination low dose cyclosporin A and steroid therapy in chronic intraocular inflammation. Eye 1990;4:514-20

3 Mochizuki M, Masuda K, Sakane T, et al. A clinical trial of FK506 in refractory uveitis. Am $\mathcal{f}$ Ophthalmol 1993;115: FK506

4 Minty A, Chalon P, Derocq JM, et al. Interleukin-13 is a new human lymphokine regulating inflammatory and immune responses. Nature 1993;362:248-50.

5 De Waal Malefyt R, Figdor CG, Huijbens R, et al. Effects of IL-13 on phenotype, cytokine production, and cytotoxic function of human monocytes. Comparison with IL-4 and modulation by IFN-gamma or IL-10. F Immunol 1993;151 6370-81

6 Doherty TM, Kastelein R, Menon S, et al. Modulation of murine macrophage function by IL-13. I Immunol 1993;151:7151-60.

7 Cosentino G, Soprana E, Thienes CP, et al. IL-13 down-regulates CD14 expression and TNF-alpha secretion in normal human monocytes. F Immunol 1995;155: 3145-51.

8 Endo T, Ogushi F, Sone S. LPS-dependent cyclooxygenase- 2 induction in human monocytes is downoxygenase-2 induction in human monocytes is down1996;156:2240-6.

9 Onoe Y, Miyaura C, Kaminakayashiki T, et al. IL-13 and IL-4 inhibit bone resorption by suppressing cyclooxygenase-2-dependent prostaglandin synthesis in osteoblasts. F Immunol 1996;156:758-64.

10 Jacobsen SE, Okkenhaug C, Veiby OP, et al. Interleukin 13 novel role in direct regulation of proliferation and differentiation of primitive hematopoieytic progenitor cells. F Exp Med 1994;180:75-82.

11 Lai YH, Heslan JM, Poppema S, et al. Continuous administration of Il-13 to mice induces extramedullary hemopoiesis and monocytosis. F Immunol 1996;156:3166-73.
12 Skeen MJ, Miller MA, Shinnick TM, et al. Regulation of murine macrophage IL-12 production. F Immunol 1996; 156:1196-206.

13 Guex-Crosier Y, Raber J, Chan C-C, et al. Humanized antibodies against the alpha-chain of the IL-2 receptor and against the beta-chain shared by the IL-2 and IL-15 receptors in a monkey uveitis model of autoimmune diseases. $\mathcal{F}$ Immunol 1997;158:452-8.

14 Fiorentino DF, Zlotnik A, Mosmann TR, et al. IL-10 inhibits cytokine production by activated macrophages. F Immunol 1991;147:3815-22.

15 SAS/STAT user's guide. Version 6. 4th ed. Cary, NC: SAS Institute Inc, 1989:891-996.

16 Marfaing-Koka A, Devergne O, Gorgone G, et al Regulation of the production of the RANTES chemokine by endothelial cells. Synergistic induction by IFN-gamma plus TNFalpha and inhibition by IL-4 and IL-13. F Immunol 1995;154:1870-8.

17 Durum S, Oppenheim JJ. Proinflammatory cytokines and immunity. In: Paul WE, ed. Fundamental immunology. New York: Raven Press, 1993:801-35.

18 Murray PI, Hoekzema R, van Haren MAC, et al. Aqueous humor interleukin-6 levels in uveitis. Invest Ophthalmol Vis Sci 1990;31:917-20.

19 Wu WC-S, Mannion B, Stone RM. Uveitis associated with interleukin-3 and interleukine-6 therapy. Arch Ophthalmol 1995;113:408-9.

20 Roberge FG, Caspi RR, Nussenblatt RB. Glial retinal Muller cells produce IL-1 activity and have a dual effect on autoimmune $\mathrm{T}$ helper lymphocytes. Antigen presentation manifested after removal of suppressive activity. I Immunol 1988;140:2193-6.

21 de Vos AF, Hoekzema R, Kijlstra A. Cytokines and uveitis, a review. Curr Eye Res 1992;11:581-97.

22 Guex-Crosier Y, Wittwer AJ, Roberge FG. Intraocular production of a cytokine (CINC) responsible for neutrophil infiltration in endotoxin-induced uveitis. $\mathrm{Br} F$ Ophthalmol 1996;80:649-52.

23 Rosenbaum JT. Towards cytokine insight in sight. $\mathrm{Br} F \mathrm{Oph}-$ thalmol 1995;79:970-1.

24 Deschenes J, Char DH, Kaleta S. Activated T lymphocytes in uveitis. Br f Ophthalmol 1988;72:83-7.

25 Chan C-C, Nussenblatt RB, Fujikawa LS, et al. Sympathetic ophthalmia. Immunopathological findings. Ophthalmology 1986;93:690-5.

26 Chan C-C, Wetzig RP, Palestine AG, et al. Immunohistopathology of ocular sarcoidosis. Report of a case and discussion of immunopathogenesis. Arch Ophthalmol 1987;105: 1398-402.

27 Chan CC, Palestine AG, Kuwabara T, et al. Immunopathological study of Vogt-Koyanagi-Harada syndrome: report of a case. Am f Ophthalmol 1988;105:607-11.

28 Vitale AT, Rodriguez A, Foster SC. Low-dose cyclosporin A therapy in treating chronic, noninfectious uveitis. Ophthalmology 1996;103:365-74.

29 Zurawski G, de Vries JE. Interleukin 13, an interleukin 4-like cytokine that acts on monocytes and B cells, but not on T cells. Immunol Today 1994;15:19-26.

30 Cash E, Minty A, Ferrara P, et al. Macrophage-inactivating IL-13 suppresses experimental autoimmune encephalomyelitis in rats. F Immunol 1994;153:4258-67. 